\title{
ROBERT LEE MOORE, 1882-1974
}

\author{
BY R. L. WILDER
}

If one were asked to list mathematicians who had the most influence on the development of American mathematics during the first half of the twentieth century, certainly R. L. Moore's name would find a prominent place among them. Among the fifty doctorates which he supervised are two former presidents of the American Mathematical Society, four former presidents of the Mathematical Association of America, three members of the National Academy of Sciences; and the number of doctorates which have originated from Moore doctorates either directly or through later generations is apparently in excess of $500 .^{2}$

These statistics already imply that the man must have been a great teacher; and that he was, any of his students would testify. The "Moore method" of teaching, the heart of which is to get the student to find his own proofs of theorems and, ultimately, to suggest and prove new theorems, has been recorded on a film made by the Mathematical Association of America with the title Challenge in the classroom. However, no film, no matter how faithful to detail, could record all the pertinent features of his teaching associated with the man's character and environment.

An essential part of the method was Moore's ability to search out and recognize creative ability among the multitude of students who presented themselves at the University of Texas. It was Moore's custom to teach five courses (which he continued to do until his retirement at age 86!) consisting of calculus, an intermediate course such as advanced calculus, and three courses which began with point set topology ("Foundations of Mathematics") and culminated in a research course. Frequently he would find a promising student in his calculus class, and from then on that student would become a major project; Moore would carry him on through one course after another of the above sequence to the $\mathrm{Ph}$.D. If any proof were needed that the capability of doing creative work in mathematics is not the rare genetic accident that it is commonly considered, Moore certainly gave it during his career as a teacher.

\footnotetext{
${ }^{1}$ For supplying information and materials, I am indebted to several of Dr. Moore's past students and colleagues, and especially to Professor Robert E. Greenwood.-R.L.W.

${ }^{2}$ A count of the doctorates listed in D. R. Traylor, Creative teaching: Heritage of R. L. Moore (University of Houston, 1972), yields 50 Moore Ph.D.'s and 442 later generations of Ph.D.'s. The latter figure is low, since apparently some of the second generation did not report their Ph.D.'s. Allowing for this fact and for the years (apparently 4) since the list was compiled, I estimate there have been over $500 \mathrm{Ph} . \mathrm{D}$ 's in the 2 nd to 6 th generations. Summarizing, I estimate over 550 "descendants" of R. L. Moore.
} 
Of course not all his students were found this way. Some were "caught" by happening to take one of his advanced courses as an elective to round out their main objectives (which were as various as chemistry, medicine, public school teaching, applied mathematics, to name but a few). Once a student with innate mathematical talent came under R. L.'s influence, his destiny was virtually decided.

Naturally, as Moore's reputation spread, advanced students from other institutions came to Texas to seek entrance to his courses. This involved careful selection on Moore's part; it can be surmised that many applied but few were chosen. Students who had already taken courses in function theory and who therefore presumably knew the basic topological properties of the linear continuum and the complex plane might be discouraged from taking Moore's course in "Foundations." And students already in "Foundations" were virtually forbidden to take courses containing related material. Reading books or papers relating to the material of the course was also ruled out. This naturally implied a relationship with other members of his department that might be very difficult to achieve in other institutions.

The aim of the "Method" was to develop research ability, not knowledge alone. To some, knowledge may mean power; but to quote a statement Moore made to a prospective student from another university, "What does information amount to compared to power?" Putting the matter in capsule form, it was a unique method employed by a unique man in a unique situation.

What kind of background nurtured such a man as R. L. Moore? He was born November 14, 1882, in Dallas, Texas, which was then virtually a frontier town. His father was a grain, hardware, and grocery merchant, and R. L. was the fifth of six children. His paternal grandfather was a Vermont physician, whose desire to study natural drugs used by the Indians and other natives of North Carolina took him for a period to that state. At the end of this period, two of his children, including R. L.'s father, decided to stay in the South (and, ultimately to support the Confederacy). R. L.'s mother was a native Virginian. It is not surprising, therefore, that $R$. L. became a "true Southerner," albeit more correctly speaking a true Texan. However, in his speech he had little, if any, of what is termed a "Texas accent." But he never lost his native Texas ideology. As a person he was proud, steadfast, and ever ready to defend his ideas, but appreciative of (often delighted with) an opponent who openly opposed him.

His strong convictions extended to his mathematical philosophy, which appeared to be strongly platonistic. The Axiom of Choice was to him a truth, a kind of mathematical absolute. Perhaps the roots of his convictions lie in the fact that he was to a considerable extent self-taught, and following his matriculation at the University of Texas in 1898 (when he was not quite 16 years old) he came under the influence of George Bruce Halsted. Halsted was an aggressive, opinionated person, but a true scholar whose somewhat indiscreet outspokenness apparently led to his leaving the University of Texas in December, 1902. Halsted was a student of Sylvester at Johns 
Hopkins. Moreover, Halsted was himself, apparently, an outstanding teacher. During his years (1884-1902) at the University of Texas his students included M. B. Porter (Ph.D., Harvard, 1897), L. E. Dickson (Ph.D., Chicago, 1896) and H. Y. Benedict (Ph.D., Harvard, 1898; later president of the University of Texas). He was also the one who, while an "instructor in post graduate mathematics" at Princeton, influenced H. B. Fine to become a mathematician. ${ }^{3}$ Probably Halsted's interest in geometry was a determining influence upon Moore. While Moore was still at Texas as a Fellow (1901-1902; see below), what can reasonably be considered his first publication (although written up by Halsted ${ }^{4}$ and not included in the Bibliography below) was a proof of the redundancy of one of Hilbert's betweenness axioms (the original Axiom II 4). This involved what sociologists call a "multiple", since E. H. Moore had published a proof shortly before. R. L.'s proof was "neater, shorter and more elegant" than E. H.'s; the latter "expressed a preference" for it, calling it "delightfully simple.",

It took R. L. only three years to earn his B.A. and M.A. degrees (both in 1901). During the year 1901-1902, he was a teaching Fellow at Texas, and during 1902-1903 he taught high school in Marshall, Texas. He then spent the years 1903-1905 as a graduate student at the University of Chicago. These years undoubtedly had a strong determinative influence on R. L., both mathematically and pedagogically. At that time, E. H. Moore, head of the Chicago department, was intensely interested in the axiomatic method, and particularly in the foundations of geometry. O. Veblen had just received (1903) his Ph.D. under E. H. Moore's supervision and had been appointed an Associate in Mathematics. Veblen's dissertation ${ }^{8}$ was devoted to his well-known system of axioms for Euclidean geometry in terms of "point" and "order." A close working relationship developed between R. L. and Veblen. The latter acknowledged R. L.'s "critically" reading the manuscript of his dissertation before its publication. Reciprocally, R. L. stated in his dissertation (after thanking both E. H. Moore and Veblen for suggestions and criticism) that Veblen not only suggested the undertaking of the investigation, but "made numerous suggestions and criticisms" as well as giving "much help in the way of actual collaboration.",

\footnotetext{
${ }^{3}$ See R. C. Archibald, A semicentennial history of the American Mathematical Society, 1888-1938, Amer. Math. Soc. Semicentennial Pub. 1 (1938), 167, 241.

${ }^{4}$ G. B. Halsted, The betweenness assumptions, Amer. Math. Monthly 9 (1902), 98-101.

${ }^{5}$ I.e., multiple discovery. As might be expected of a prolific mathematician, R. L. Moore was involved in several multiples.

${ }^{6}$ The quotes are from the faculty memorial, In Memoriam, Robert Lee Moore, prepared by a special committee of Moore's colleagues and presented to the faculty of the University of Texas.

${ }^{7}$ This quote is from a note in Amer. Math. Monthly, 9 (1902), 152-153.

${ }^{8}$ O. Veblen, A system of axioms for geometry, Trans. Amer. Math. Soc. 5 (1904), 343-384.

${ }^{9}$ Due to the lack of adequate records, disagreement exists concerning who was the "official" director of R. L.'s doctoral dissertation. In his article on R. L. cited in footnote 3, R. C. Archibald states that R. L.'s dissertation was done under Veblen's direction (loc. cit., p. 209), and others have confirmed this. There can be little question, however, that E. H. Moore was the prime guiding influence of both Veblen and R. L.
} 
E. H. Moore's influence on R. L.'s teaching methods was probably great. Those familiar with E. H.'s mode of teaching assert that they detect elements of the method later developed by R. L.

After leaving Chicago, R. L. taught a year at the University of Tennessee at Knoxville. He then spent the years 1906-1908 teaching at Princeton, the years 1908-1911 at Northwestern, and moved to the University of Pennsylvania in 1911, where he spent the next nine years. During his stay at Pennsylvania, Moore directed his first three doctoral dissertations including that of J. R. Kline, who was later to become, for many years, Secretary of the American Mathematical Society.

Moore began his service with the University of Texas in 1920, where he continued teaching until he reached the official retirement age of 70 in 1951. $\mathrm{He}$ was then "named... Professor of Mathematics and Astronomy on Modified Service. Due to a time lag in the establishment of retirement policies for public servants in Texas, the University of Texas continued staff members beyond age 70 on half-time pay and half-time service. But R. L. Moore was still vigorous and he disdained the word retirement. He continued to teach his full load of five organized courses but by law was allowed only half-time pay. He would not break the habit of teaching a complete sequence of courses each year, beginning with the calculus;" ${ }^{10}$ and he continued teaching until 1969. It is notable that early in his career at Texas, he was appointed The University Research Lecturer for the year 1929. He was elected to the National Academy of Sciences in 1931.

Among his services for the American Mathematical Society were: Associate Editor of the Transactions, 1914-1927; Vice President, 1923; Colloquium Lecturer, 1929; Committee on Colloquium Publications, 1929-1936 (chairman 1930-1933); Visiting Lecturer, 1931-1932; President, 19371938.

He was married to Margaret MacLellan Key in 1910. She survives him. ${ }^{10 a}$ It can only be surmised how much her strong devotion to R. L. and to his work contributed to his success.

\section{Scientific Work}

GEOMETRY. The work in geometry was mainly devoted to the axiomatic foundations. Bibliography Items 1 and 2 were originally presented to the Society (April 22, 1905) under the same title, Sets of metrical hypotheses for geometry. Item 1 shows that while Dehn proved that Hilbert's original axiom sets I, II and IV, augmented by the assertion, S, that the sum of the angles of a triangle is two right angles, are not sufficient to yield III (parallels), nevertheless any space satisfying them (i.e., I, II, IV, S) must be a part of a space $^{11}$ in which III holds. Item 2 was Moore's dissertation, and gives axioms for Euclidean geometry based on the primitive notions of point, order, and

\footnotetext{
${ }^{10}$ The quotation is from the faculty memorial cited in footnote 6 .

${ }^{10 a}$ Mrs. Moore died December 18, 1975.

${ }^{11}$ I.e., by the addition of ideal points.
} 
congruence, together with various alternatives. It is closely related to Veblen's dissertation, ${ }^{8}$ whose axioms I, and III-X it ultilizes. Item 3 is directed specifically at modification of Veblen's axioms.

Item 5 is important for at least two reasons: (1) It shows that the space $V$ satisfying axioms I-VIII of Veblen's thesis, and for which Veblen gave his proof of the Jordan Curve Theorem (published 10 years before) ${ }^{12}$ is actually metrizable, in that $V$ is topologically equivalent to the euclidean coordinate plane. (2) It indicates the topological direction in which the major part of Moore's future work was to lie. Item 17 is a thorough-going point set analysis of the euclidean and Bolyai-Lobachewskian planes in terms of point, region, and motion. It was inspired by Hilbert's Über die Gundlagen der Geometrie, ${ }^{13}$ and both the first six axioms and the methods are reminiscent of Item 10 It differs from Hilbert's treatment in that it simultaneously analysis of the euclidean and Bolyai-Lobachewskian planes in terms of whereas Hilbert's analysis is largely confined to the group.

The review (Item 18) of the second volume of the Veblen-Young ${ }^{14}$ work on projective geometry is mostly devoted to a critical analysis of its foundations, particularly as to whether a certain one of the defined terms should not really be an undefined term. This seems to be the only review written by Moore; possibly the amount of time which he must have spent on it discouraged him from undertaking further reviews. One in whom the faculty for precision and logical structure is so well developed can usually find flaws in any book which will afford a challenge to make improvements.

Foundations of ANALYsis. In this category we put Items 4, 6, 7, 16, 30, 34 and 42 . This is perhaps rather arbitrary, since all Moore's papers in point set theory bear relations, either direct or indirect, to analysis. ${ }^{15}$ In Item 4 he proposed a form of Duhamel's theorem suitable to wider application than the form previously given by Osgood, and in Items 6 and 7 he gave sets of axioms in terms of point and limit for the linear continuum. Item 16 gives necessary and sufficient conditions for a certain type of Fréchet space to be compact (in the covering sense), and Item 25 disproves a proposition stated by E. W. Hobson in his classic Theory of functions of a real variable. Item 30 is concerned with the relatively uniform convergence introduced by $\mathrm{E}$. $\mathrm{H}$. Moore, specifically with regard to functions defined on a measurable set.

POINT SET THEORY. In deference to Moore's own preferences, we use this term even though many would use the term set-theoretic topology. Although, as noted above, signs of Moore's proceeding in this direction are to be found in his earlier work, they erupted full-blown in Item 10 (On the foundations of plane analysis situs); Item 9 is a preliminary announcement of Item 10 . Presented therein are three systems of axioms $\Sigma_{1}, \Sigma_{2}, \Sigma_{3}$, all based on point and region as primitive terms.

\footnotetext{
${ }^{12} \mathrm{O}$. Veblen, Theory of plane curves in non-metrical analysis situs, Trans. Amer. Math. Soc. 6 (1905), 83-98.

${ }^{13}$ Math. Ann. 56 (1902-1903), 381-422.

${ }^{14}$ This second volume was actually written by Veblen alone.

${ }^{15}$ Moore called his beginning course in point set theory "Foundations of Mathematics."
} 
An outstanding feature of $\Sigma_{1}$ and $\Sigma_{2}$ is an axiom (Axiom 1) which, in later terminology, implies regularity and existence of a countable base. This was well in advance of the work of Urysohn and others on metrization theorems in terms of these properties. System $\Sigma_{3}$ does not imply metrizability, Axiom 1 being replaced by an axiom assuming only a countable base at each point. For a number of years both Moore and his students who had taken positions at other universities, used the system $\Sigma_{1}$ as a basis for teaching their beginning courses in topology by the "Moore Method" (or a suitable approximation thereto). The chief stumbling block in this course for students was Theorem 15 to the effect that every connected open set is arcwise connected. Indeed, one might say that the ability to prove this theorem was sort of an "open sesame" to Ph.D. candidacy. And it was well known among the graduates of the "Moore School" that if one wanted to get a student into one of the universities at which Moore students were teaching, all that was needed was to be able to say of him, "He proved Theorem 15." (In later years, however, Moore used a new system based on axioms given in his colloquium book, Item 51.) In Item 14, Moore showed that every space that satisfied either $\Sigma_{1}$ or $\Sigma_{2}$ is topologically equivalent to a number plane. ${ }^{16}$

Moore was to return later to the axiomatic characterization of the plane (2-sphere) and its topology in both his book (Item 51) and Item 53; in the latter the undefined terms were place (which may be interpreted as bounded, connected, open set) and a relation imbedded in.

Item 11, A theorem concerning continuous curves, published in 1917 , is the first of a large literature on continuous curves (= locally connected, metric continua) to emanate from Moore and his school. It was possibly inspired by the well-known Hahn-Mazurkiewicz topological characterization of the continuous curve defined analytically in Jordan's Cours d'analyse and proves the arcwise connectivity of such configurations. ${ }^{17}$

The report on continuous curves which Moore gave at a symposium in Lawrence, Kansas in 1922, Item 27, covers the results which had been obtained up to this time so far as the topological nature of these curves was concerned. It is important not simply as a summary of results, but as indicating lines of research which were to be followed up by his own school (especially G. T. Whyburn ${ }^{18}$ ) and others. Except for such items as 26, 44 and 45, however, and occasional theorems in other papers, Moore left the further investigation of continuous curves to his students, while concentrating himself on problems concerning general point sets, especially continua.

In discussing the rest of Moore's publications in point set theory, it will be convenient to divide them into two classes, viz. structural and positional. By

\footnotetext{
${ }^{16}$ That the eight axioms of $\Sigma_{1}$ may be reduced to seven was later shown in R. L. Wilder, Concerning R. L. Moore's axioms $\Sigma_{1}$ for plane analysis situs, Bull. Amer. Math. Soc. 34 (1928), 752-760.

${ }^{17}$ This turned out to be another "multiple" in which Moore was involved, in that it was independently proved by both S. Mazurkiewicz and $\mathrm{H}$. Tietze.

${ }^{18}$ See, for instance, B. L. McAllister, Cyclic elements in topology, a history, Amer. Math. Monthly 73 (1966), 337-350.
} 
"structural" we indicate internal properties, and by "positional" we indicate relations with an imbedding space.

Structural papers. Items 28 and 31 extend a theorem of Sierpiński (Item 31 turned out to be a "multiple" with Mazurkiewicz; cf. footnote 8 of Item 37) and Item 39 duplicated earlier results of W. Gross and Fréchet. Item 41 was a contribution to the theory of Indecomposable continua, a type of configuration which received much attention from topologists in both the United States and Poland. The title of Item 43 is self-explanatory.

Items 54-56 are of particular interest in that they display a system of axioms whose primitive terms, in addition to point and region, contain the term contiguous to, denoting a relation between points. In particular one point can be contiguous to another. Presumably a major reason for introducing this notion was for its application to structural properties of a continuum in terms of specialized subsets; for example, if the cyclic elements of a continuous curve $C$ are regarded as "points" and two such points $p$ and $q$ are called contiguous if and only if one of the pair $p, q$ is a point (in the ordinary sense) of the other, then $C$ becomes an acyclic continuous curve in terms of its "points." It is perhaps curious that this material has not created more subsequent research than it has, since certainly the notion of contiguous point should prove fruitful, not only as a mathematical concept, but as a physical notion. $^{19}$

Items 57, 59, and 64 continue Moore's researches into the structure of continua, making special use of such concepts as continua of condensation and upper semicontinuous collections of continua. Upper semicontinuous collections were introduced in Item 38, where it was shown that if such a collection, $G$, of mutually exclusive bounded continua fills up a plane $E$ and none of its elements separates $E$, then it is itself a plane in terms of the elements of $G$ as "points" and with "limit point" suitably defined. A similar theorem holds true for the 2 -sphere, $S$, and in Item 50, Moore showed that if the elements of $G$ are allowed to separate $S$, then the resulting configuration, $C$, is a cactoid ( $=$ a continuous curve whose maximal cyclic elements are 2-spheres). In view of the prescribed definition of limit for the elements of an upper semicontinuous collection, these elements are the counterimages of points of $C$ under a monotonic continuous mapping of $S$ onto $C$. This theorem was not only generalized to 2-manifolds and higher dimensional configurations, ${ }^{20}$ but the notion of monotone mapping proved very fruitful in later set-theoretic investigations.

The notion of triod was introduced in Items 46 and 49. One of the most

${ }^{19}$ Cf. T. Hailperin, On contiguous point spaces, Bull. Amer. Math. Soc. 45 (1939), 172-174; E. C. Klipple, Two-dimensional spaces in which there exist contiguous points, Trans. Amer. Math. Soc. 41 (1938), 250-276; and K. S. Butcher, A homology theory for multiply connected contiguous point spaces, Univ. of Michigan Dissertation, 1946.

${ }^{20}$ Cf. J. H. Roberts and N. E. Steenrod, Monotone transformations of two-dimensional manifolds, Ann. of Math. 39 (1938), 851-862; R. L. Wilder, Monotone mappings of manifolds, Pacific J. Math. 7 (1957), 1519-1523, and Monotone mappings of manifolds. II, Michigan Math. J. 9 (1958), 19-23. 
striking results was the impossibility of imbedding an uncountable number of disjoint triods in the plane.

Prime part decompositions, which had been introduced by $\mathrm{H}$. Hahn, were exploited in Items 29 and $35 .^{21}$ It was shown, for instance, that in terms of its prime parts, every bounded continuum is a continuous curve (possibly degenerate). The prime part notion was further extended and generalized by G. T. Whyburn and the present writer.

POSITIONAL PAPERS. Item 12, A characterization of Jordan regions by properties having no reference to their boundaries, (1918), was evidently principally inspired by (1) A. Schoenflies' classic work Die Entwickelung der Lehre von den Punktmannigfaltigkeiten (Zweiter Teil, Leipzig, Teubner, 1908) in which, among other results concerning positional properties of plane continuous curves, conditions were given under which the common boundary of two plane domains will be a simple closed curve, and (2) Caratheodory's work on prime ends. Like Caratheodory's condition, given for a like purpose, Moore's condition of "uniform connectedness im kleinen" applied to one domain alone; otherwise it is much simpler than the Caratheodory condition, and in the higher dimensional properties "ulc $n$ " and "ULC $n$ " has led to extensive generalizations. Item 21 gives examples in three-dimensional space for which neither Moore's theorem nor the theorem of Schoenflies holds.

In earlier work of Zoretti, F. Riesz, Schoenflies and Denjoy, it developed that every closed, bounded, totally disconnected plane point set is a subset of an arc. In Item 15, written jointly with J. R. Kline, ${ }^{22}$ necessary and sufficient conditions were given in order that a plane point set should be a subset of an arc. (This was later extended to $n$-dimensional space by E. W. Miller (On subsets of a continuous curve which lie on an arc of the continuous curve, Amer. J. Math. 54 (1932), 397-416).)

The concept of equicontinuous systems of curves was introduced in Item 20 , and in Item 24 was used to characterize both closed 2-cells and open surfaces in three-dimensional space. "Property S," a property weaker than uniform local connectedness yet stronger than local connectedness, was introduced in Item 22; a modification of a notion that Sierpiński had used to characterize continuous curves, it was used here to characterize those simply connected plane domains which have continuous curve boundaries. And with reference to bounded plane domains that are complementary to continuous curves, Moore proved in Item 23 that their outer boundaries are simple closed curves; from this he was able to show that if two points are separated by a continuous curve, $C$, they they are separated by a simple closed curve of $C$.

Spirals were introduced (in the plane) in Item 68 and certain results established concerning sets of points on which a spiral may close down; e.g.,

\footnotetext{
${ }^{21}$ Item 29 contains certain errors whose corrections were indicated in the footnote at the bottom of pp. $426-427$ of Item 38 .

${ }^{22}$ This seems to be the only jointly authored paper in which Moore was involved.
} 
if $M$ is a compact, totally disconnected point set and $p$ is a point not in $M$, then there exists an arc from $p$ which spirals down on every point of $M$ but on no point that is not in $M$. Several of Moore's later doctoral students found further results concerning this notion.

Others of his positional papers, as their titles indicate, treat plane separation and accessibility. Item 44 establishes an interesting theorem to the effect that any two points in the complement of a plane continuous curve $M$ can be joined by an arc that does not separate $M$.

Moore's book, Foundations of point set theory, published in 1932 and, in revised form, in 1962 by the American Mathematical Society as Volume 13 in the Colloquium Publications Series, is based on the colloquium lectures which he gave before the Society in Boulder, Colorado, in August, 1929. The entire treatment, as might be expected, is based on an axiom system whose primitive terms are point and region. In a way reminiscent of his fundamental 1916 paper (Item 10), it does not, however, use his original Axiom 1, but presents a new axiom presumably designed to accomplish most of the purposes of the original, but without implying that the space is metrizable or separable. On the basis of this axiom (plus an axiom which states that every region is a point set), he proves 183 theorems in the first chapter alone. Additional axioms are added in subsequent chapters, but not until the last few pages of the book are axioms added which will ensure that the space is a plane or 2-sphere. Consequently the book is a very general compendium of point set results which has served, among other functions, as a reference point for many subsequent investigations.

\section{Epilogue}

Although Moore's teaching and research have been treated separately above, in practice there was no true separation. His own research results formed the basis of his teaching, and if they had no other justification, their use in this wise would have been sufficient. It is cause for conjecture just how much of his success as a teacher was due to his bringing his own ideas into class and allowing his students to participate in a reenactment of their creation; surely this experience must have created an empathy which the use of other materials, even using the "Method," would not have engendered.

It is pleasant to relate that the University of Texas has recognized his contributions by the dedication, in 1973, of a new 17 level, double-winged building housing the departments of Astronomy, Mathematics and Physics, now known as Robert Lee Moore Hall.

\section{BIBLIOGRAPHY}

1. Geometry in which the sum of the angles of every triangle is two right angles, Trans. Amer. Math. Soc. 8 (1907), 369-378.

2. Sets of metrical hypotheses for geometry, Trans. Amer. Math. Soc. 9 (1908), 487-512.

3. A note concerning Veblen's axioms for geometry, Trans. Amer. Math. Soc. 13 (1912), 74-78.

4. On Duhamel's theorem, Ann. of Math. 13 (1912), 161-168. 
5. On a set of postulates which suffice to define a number-plane, Trans. Amer. Math. Soc. 16 (1915), 27-32.

6. The linear continuum in terms of point and limit, Ann. of Math. 16 (1915), 123-133.

7. On the linear continuum, Bull. Amer. Math. Soc. 22 (1915), 117-122.

8. Concerning a non-metrical pseudo-Archimedean axiom, Bull. Amer. Math. Soc. 22 (1916), 225-236.

9. On the foundations of plane analysis situs, Proc. Nat. Acad. Sci. U.S.A. 2 (1916), 270-272.

10. On the foundations of plane analysis situs, Trans. Amer. Math. Soc. 17 (1916), 131-164.

11. A theorem concerning continuous curves, Bull. Amer. Math. Soc. 23 (1917), 233-236.

12. A characterization of Jordan regions by properties having no reference to their boundaries,

Proc. Nat. Acad. Sci. U.S.A. 4 (1918), 364-370.

13. Continuous sets that have no continuous sets of condensation, Bull. Amer. Math. Soc. 20 (1919), 174-176.

14. Concerning a set of postulates for plane analysis situs, Trans. Amer. Math. Soc. 20 (1919), 169-178.

15. (With J. R. Kline) On the most general plane closed point set through which it is possible to pass a simple continuous arc, Ann. of Math. 20 (1919), 218-223.

16. On the most general class L of Fréchet in which the Heine-Borel-Lebesgue theorem holds true, Proc. Nat. Acad. Sci. U.S.A. 5 (1919), 206-210.

17. On the Lie-Riemann-Helmholtz-Hilbert problem of the foundations of geometry, Amer. J. Math. 41 (1919), 299-319.

18. The second volume of Veblen and Young's projective geometry, Bull. Amer. Math. Soc. 26 (1920), 412-425 (book review).

19. Concerning simple continuous curves. Trans. Amer. Math. Soc. 21 (1920), 333-347.

20. Concerning certain equicontinuous systems of curves, Trans. Amer. Math. Soc. 22 (1921), 41-45.

21. On the relation of a continuous curve to its complementary domains in space of three dimensions, Proc. Nat. Acad. Sci. U.S.A. 8 (1922), 33-38.

22. Concerning connectedness im kleinen and a related property, Fund. Math. 3 (1922), 232-237.

23. Concerning continuous curves in the plane, Math. Z. 15, (1922), 254-260.

24. On the generation of a simple surface by means of a set of equicontinuous curves, Fund. Math. 4 (1923), 106-117.

25. An uncountable, closed and non-dense point set each of whose complementary intervals abuts on another one at each of its ends, Bull. Amer. Math. Soc. 29 (1923), 49-50.

26. Concerning the cut-points of continuous curves and of other closed and connected pointsets, Proc. Nat. Acad. Sci. U.S.A. 9 (1923), 101-106.

27. Report on continuous curves from the viewpoint of analysis situs, Bull. Amer. Math. Soc. 29 (1923), 289-302.

28. An extension of the theorem that no countable point set is perfect, Proc. Nat. Acad. Sci. U.S.A. 10 (1924), 168-170.

29. Concerning the prime parts of certain continua which separate the plane, Proc. Nat. Acad. Sci. U.S.A. 10 (1924), 170-175.

30. Concerning relatively uniform convergence, Bull. Amer. Math. Soc. 30 (1924), 504-505.

31. Concerning the sum of a countable number of mutually exclusive continua in the plane, Fund. Math. 6 (1924), 189-202.

32. Concerning upper semi-continuous collections of continua which do not separate a given continuum, Proc. Nat. Acad. Sci. U.S.A. 10 (1924), 356-360.

33. Concerning the common boundary of two domains, Fund. Math. 6 (1924), 203-213.

34. Concerning sets of segments which cover a point set in the Vitali sense, Proc. Nat. Acad.

Sci. U.S.A. 10 (1924), 464-467.

35. Concerning the prime parts of a continuum, Math. Z. 22 (1925), 307-315.

36. A characterization of a continuous curve, Fund. Math. 7 (1925), 302-307.

37. Concerning the separation of point sets by curves, Proc. Nat. Acad. Sci. U.S.A. 11 (1925), 469-476. 
38. Concerning upper semi-continuous collections of continua, Trans. Amer. Math. Soc. 27 (1925), 416-428.

39. Concerning the relation between separability and the proposition that every uncountable point set has a limit point, Fund. Math. 8 (1926), 189-192; cf., also, An acknowledgement, ibid., 374-375.

40. Conditions under which one of two given closed linear point sets may be thrown into the other one by a continuous transformation of a plane into itself, Amer. J. Math. 48 (1926), 67-72.

41. Concerning indecomposable continua and continua which contain no subsets that separate the plane. Proc. Nat. Acad. Sci. U.S.A. 12 (1926), 359-363.

42. Covering theorems, Bull. Amer. Math. Soc. 32 (1926), 275-282.

43. A connected and regular point set which contains no arc, Bull. Amer. Math. Soc. 32 (1926), 331-332.

44. Concerning paths that do not separate a given continuous curve, Proc. Nat. Acad. Sci. U.S.A. 12 (1926), 745-753.

45. Some separation theorems, Proc. Nat. Acad. Sci. U.S.A. 13 (1927), 711-716.

46. Concerning triods in the plane and the junction points of plane continua, Proc. Nat. Acad. Sci. U.S.A. 14 (1928), 85-88.

47. On the separation of the plane by a continuum, Bull. Amer. Math. Soc. 34 (1928), 303-306.

48. A separation theorem, Fund. Math. 12 (1928), 295-297.

49. Concerning triodic continua in the plane, Fund. Math. 13 (1929), 261-263.

50. Concerning upper semi-continuous collections, Monatsh. Math. Phys. 36 (1929), 81-88.

51. Foundations of point set theory, Amer. Math. Soc. Colloq. Publ., vol. 13, Amer. Math. Soc., Providence, R. I., 1932; rev. ed., 1962 (Large-sized page format in revised edition); reprinted with corrections, 1970. MR 27 \#709.

52. Concerning compact continua which contain no continuum that separates the plane, Proc.

Nat. Acad. Sci. U.S.A. 20 (1934), 41-45.

53. A set of axioms for plane analysis situs, Fund. Math. 25 (1935), 13-28.

54. Foundations of a point set theory of spaces in which some points are contiguous to others, Rice Institute Pamphlet 23 (1936), 1-41.

55. Upper semi-continuous collections of the second type, Rice Institute Pamphlet 23 (1936), $42-57$.

56. On the structure of continua, Rice Institute Pamphlet 23 (1936), 58-74.

57. Concerning essential continua of condensation, Trans. Amer. Math. Soc. 42 (1937), 41-52.

58. Concerning accessibility, Proc. Nat. Acad. Sci. U.S.A. 25 (1939), 648-653. MR 1, 107.

59. Concerning the open subsets of a plane continuum, Proc. Nat. Acad. Sci. U.S.A. 26 (1940), 24-25. MR 1, 107.

60. Concerning separability, Proc. Nat. Acad. Sci. U.S.A. 28 (1942), 56-58. MR 3, 136.

61. Concerning intersecting continua, Proc. Nat. Acad. Sci. U.S.A. 28 (1942), 544-550. MR 4, 146.

62. Concerning a continuum and its boundary, Proc. Nat. Acad. Sci. U.S.A. 28 (1942), 550-555. MR 4, 146.

63. Concerning domains whose boundaries are compact, Proc. Nat. Acad. Sci. U.S.A. 28 (1942), 555-561. MR 4, 146.

64. Concerning continua which have dendratomic subsets, Proc. Nat. Acad. Sci. U.S.A. 29 (1943), 384-389. MR 5, 149.

65. Concerning webs in the plane, Proc. Nat. Acad. Sci. U.S.A. 29 (1943). 389-393. MR 5, 150.

66. Concerning tangents to continua in the plane, Proc. Nat. Acad. Sci. U.S.A. 31 (1945), 67-70. MR 6, 181.

67. A characterization of a simple plane web, Proc. Nat. Acad. Sci. U.S.A. 32 (1946), 311-316. MR 8, 333.

68. Spirals in the plane, Proc. Nat. Acad. Sci. U.S.A. 39 (1953), 207-213. MR 14, 783. 